\title{
PRINCIPAIS PATOLOGIAS EM IDOSOS: AÇÃO EDUCATIVA E REFLEXIVA COM ADOLESCENTES
}

\author{
MAIN PATHOLOGIES IN ELDERLY: EDUCATIONAL AND \\ REFLECTIVE ACTION WITH ADOLESCENTS
}

\author{
Amanda Haissa Barros Henriques ${ }^{1}$ \\ Rayrla Cristina de Abreu Temoteo ${ }^{2}$ \\ Sthephanie de Abreu Freitas ${ }^{3}$ \\ Ariadne Pereira Pedroza ${ }^{4}$ \\ Francisco Ronner Andrade da Silva ${ }^{5}$
}

RESUMO: Objetivo: Relatar a experiência vivenciada por uma ação do Projeto de Extensão com jovens escolares a respeito das principais patologias que acometem os idosos. Método: Trata-se de um estudo descritivo, do tipo Relato de Experiência, a partir da realização de uma ação educativa sobre as principais patologias na terceira idade, na execução do Projeto de Extensão intitulado 'Educação em Saúde para Jovens Escolares: o processo de Envelhecimento em Foco', desenvolvido durante os meses de junho a novembro de 2018. Resultados: Enfatizou- se, ao longo da ação, a troca de informações sobre as principais patologias na pessoa idosa, como por exemplo, Câncer, Infarto Agudo Miocárdio, Doença de Alzheimer, Doença de Parkinson, Osteoporose, Incontinência Urinária. Por meio de slides e peça teatral, os adolescentes deviam acertar sobre qual doença se tratava a encenação. Após cada pergunta, os extensionistas abordavam fatores de riscos e

\footnotetext{
1 Mestre em Enfermagem pelo Programa de Pós-graduação Associado em Enfermagem da Universidade do Pernambuco e Universidade Estadual da Paraíba (UPE/UEPB). Docente do Curso Técnico em Cuidados de Idosos do Instituto Federal de Educação, Ciência e Tecnologia da Paraíba (IFPB) campus Mangabeira - PB, E- mail: amandahaissa@gmail.com.

2 Doutoranda em Enfermagem pelo Programa de Pós-graduação em Enfermagem da Universidade Federal do Rio Grande do Norte (UFRN). Docente do Curso Técnico em Cuidados de Idosos do Instituto Federal de Educação, Ciência e Tecnologia da Paraíba (IFPB) campus Mangabeira - PB, Email: rayrlacz@hotmail.com.

${ }^{3}$ Mestre em Enfermagem pelo Programa de Pós-graduação em Enfermagem da Universidade Federal da Paraíba (UFPB). Orientadora educacional na Editora Brasileiro e Passos. E-mail: stheenf@gmail.com.

${ }^{4}$ Mestre em Enfermagem pelo Programa de Pós-graduação em Enfermagem da Universidade Federal da Paraíba (UFPB). Docente da Faculdade São Francisco da Paraíba (FASP). Enfermeira assistencial no Hospital Universitário Júlio Bandeira (HUJB). E-mail: ariadne.pedroza@gmail.com.

${ }_{5}^{5}$ Mestre em Terapia Intensiva. Docente dos Curso de Graduação em Saúde da Faculdade São Francisco da Paraíba (FASP). E-mail: ronner_andrade@hotmail.com
} 
causas para as referidas doenças e sinais e sintomas, objetivando que ainda na juventude os alunos possam preveni-las, para viver a velhice da forma mais saudável e com qualidade, deixando como pergunta reflexiva para os mesmos: 0 que estou fazendo para prevenir estas doenças? Conclusão: Evidenciou-se o empoderamento de cerca de 200 jovens beneficiados sobre as principais patologias em idosos e a promoção da saúde ainda na juventude, reforçando a importância do processo educativo como parte fundamental do entendimento de jovens escolares no contexto do Envelhecimento e de como prevenir algumas doenças ainda na juventude para vivenciar uma velhice saudável.

Descritores: Educação em saúde. Envelhecimento. Idoso. Doenças.

ABSTRACT: Objective: To report the experience of an action of the Extension Project with young schoolchildren regarding the main pathologies that affect the elderly. Method: This is a descriptive study, of the Experience Report type, based on the realization of an educational action on the main pathologies in the third age, in the execution of the Extension Project entitled 'Health Education for Young Schoolchildren: the process of Aging in Focus', developed during the months of June to November 2018. Results: During the action, the exchange of information on the main pathologies in the elderly was emphasized, such as, for example, Cancer, Acute Myocardial Infarction, Disease Alzheimer's disease, Parkinson's disease, Osteoporosis, Urinary incontinence. Through slides and a play, the adolescents were supposed to be right about which illness the staging was about. After each question, the extension agents addressed risk factors and causes for the aforementioned diseases and signs and symptoms, aiming that even in their youth, students can prevent them, to live old age in the most healthy and quality way, leaving as a reflective question for the same: What am I doing to prevent these diseases? Conclusion: The empowerment of about 200 young people benefited over the main pathologies in the elderly and the promotion of health in youth was evidenced, reinforcing the importance of the educational process as a fundamental part of the understanding of young students in the context of Aging and how to prevent some diseases still in their youth to experience a healthy old age.

Descriptors: Health education. Aging. Elderly. Diseases. 


\section{INTRODUÇÃO}

A transição demográfica ocasionada pelo aumento da expectativa de vida dos brasileiros vem crescendo e ganhando destaque nos últimos anos. O processo de envelhecimento da população vem despertando interesse para as peculiaridades dessa faixa etária, atentando para suas necessidades assistenciais à saúde, sociais e econômicas. O envelhecimento populacional torna-se, então, um dos maiores desafios da saúde pública contemporânea (CAMARGOS; GONZAGA, 2015; QUEIROZ et al., 2012).

Este processo possui diversas particularidades e pode ser acompanhado por incapacidades geradas em decorrência das diversas alterações no organismo e pelo declínio da funcionalidade dos sistemas do indivíduo, acarretando desde comprometimento na execução das atividades diárias do idoso até o óbito (CORDEIRO et al., 2014; MORAES, 2012).

O processo de envelhecimento pode ser determinado pela perda funcional progressiva que ocorre de forma gradual, universal e irreversível com o avançar da idade (CAMPOLINA et al., 2013). Segundo Caporicci e Neto (2011), o processo de envelhecimento não pode ser definido apenas pela sua idade cronológica; outros fatores como as condições físicas, funcionais, mentais, entre outros, podem influenciar diretamente sobre esse processo, o que caracteriza que 0 envelhecimento é algo individualizado que difere de pessoa para pessoa. Estes, somados aos fatores ambientais, imprimem uma visão biopsicossocial ao envelhecimento.

Dessa forma, não se pode ignorar o indivíduo que está em processo de envelhecimento ou que já envelheceu. A sociedade precisa mudar sua conduta em relação à velhice, pois uma sociedade que tem consciência dos direitos dos indivíduos que conseguem envelhecer é capacitada para conduzir mobilizações que garantam o espaço social dos idosos. 
Quanto mais se discutir sobre questões gerontológicas e as principais alterações no organismo dos idosos, na formação de jovens escolares, por exemplo, maior empoderamento os mesmos terão para enfrentar possíveis dificuldades com o público idoso, bem como, maior entendimento e crescimento pessoal para lidar com as diferenças, limitações e particularidades de um grupo populacional que só tende a crescer cada vez mais diante do aumento da expectativa de vida (LEITE et al., 2014; PINTO et al., 2013).

Espera-se com este estudo contribuir para uma sociedade baseada em valores francos e positivos no contexto do Envelhecimento, com ênfase no entendimento de alterações no organismo que geram limitações aos idosos, bem como, com respeito às diferenças entre as faixas etárias no mundo atual, onde todas as épocas e gerações tiveram seus momentos, e para o bem ou para o mal, envelhecer é parte inexorável daqueles que não se vão, é dávida alcançada, devendo, com isso, ser respeitado e compreendido da melhor forma possível, inclusive pelos jovens.

Objetivou-se relatar a experiência vivenciada por uma ação educativa do Projeto de Extensão com jovens escolares a respeito das principais patologias que acometem os idosos, visando empoderá-los sobre a promoção da saúde dos mesmos ainda na juventude, visando um envelhecimento futuro de forma saudável através de bons hábitos de vida.

\section{MÉTODO}

Trata-se de um estudo com delineamento descritivo, do tipo Relato de Experiência, a partir da realização de uma ação educativa sobre as principais patologias que acometem os idosos. Esta ação fez parte do plano de execução do Projeto de Extensão intitulado 'Educação em Saúde para Jovens Escolares: o processo de Envelhecimento em Foco', o qual fora desenvolvido durante os meses de junho a novembro de 2018. 
Tal ação foi realizada por discentes e docentes do Curso Técnico Subsequente em Cuidados de Idosos, IFPB Campus Mangabeira, com jovens escolares de turmas do $1^{\circ}$ ano de Ensino Médio da Escola Técnica Estadual Pastor João Pereira Gomes Filho, João Pessoa-PB, visando o empoderamento dos jovens sobre o processo de envelhecimento ainda na juventude para vivenciar e respeitar a velhice, especificamente enfatizando a temática voltada para as principais patologias em idosos e a reflexão sobre o que podemos fazer na juventude para se ter uma velhice mais saudável.

$\mathrm{Na}$ discussão sobre o processo de Envelhecimento as escolas tornam-se um espaço de reflexão, pois possibilitam aos adolescentes agregarem valores que carregarão para o resto de suas vidas, assim como, compartilharem essas reflexões nos ambientes onde convivem. À medida que se realizavam as reuniões de preparo para a execução da ação e ação, propriamente dita, utilizava-se a técnica de observação e a descrição de todas as atividades desenvolvidas em um diário de campo. Tais registros culminaram na elaboração do relatório final do Projeto de Extensão, assim como na construção deste artigo.

\section{RESULTADOS E DISCUSSÃO}

O presente relato de experiência refere-se a uma ação educativa sobre as principais patologias que acometem os idosos. Esta ação fora desenvolvida durante execução do Projeto de Extensão intitulado 'Educação em Saúde para Jovens Escolares: o processo de Envelhecimento em Foco', realizado durante os meses de junho a novembro de 2018, com o objetivo de implementar ações de Educação em Saúde com jovens escolares a respeito do processo de Envelhecimento e todo seu contexto. O projeto teve como público-alvo os jovens das quatro turmas do $1^{\circ}$ Ano do Ensino Médio na Escola Técnica Estadual Pastor João Pereira Gomes Filho, João Pessoa- PB, totalizando aproximadamente, 200 adolescentes.

Para execução da ação, a princípio, realizou-se reuniões com a coordenadora geral do projeto, Docentes, Bolsistas e Voluntários, com intuito de apresentar a 
proposta da atividade educativa, seu objetivo e discussão acerca do tema 'Principais Doenças que acometem os Idosos', com base nos hábitos de vida que buscamos durante a juventude para prevenir tais patologias na velhice.

Nas reuniões da equipe executora, preparou-se todo o material a ser utilizado, como por exemplo, slides, vídeo, roteiro, dinâmica, convite para a ação, bem como a confecção das placas de identificação das turmas e os coletes para os representantes das mesmas, sendo estas identificadas por cores diferentes. Os discentes extensionistas também receberam material de apoio para leitura e embasamento da temática trabalhada.

Em forma de Gincana, as turmas escolhiam um representante, bem como o grito de guerra que os representavam, pontuando em um Quadro Placar ao longo das ações do Projeto de Extensão, que contou com 6 ações educativas ao total, sendo revelada a equipe vencedora após contabilização dos pontos.

Em se tratando desta ação, propriamente dita, cujo tema foi 'Principais Doenças que acometem os Idosos', a mesma teve início com algumas indagações e um vídeo explicativo sobre as principais doenças que o idoso apresenta, o que gerou uma discussão inicial a respeito do tema.

Para trabalhar a temática, escolheu-se doenças mais comuns e frequentes nos dias de hoje, como por exemplo, Câncer, Infarto Agudo Miocárdio, Doença de Alzheimer, Doença de Parkinson, Osteoporose, Incontinência Urinária, enfatizando os respectivos sinais e sintomas das mesmas, bem como formas de preveni-las e os impactos que apresentam na velhice.

Os extensionistas representaram vários idosos, por meio de peça teatral, cada um, por vez, descrevendo sintomas e características a respeito das doenças citadas. Após a dramatização, as equipes deviam acertar sobre qual doença se tratava a encenação, mediante as alternativas proposta nos slides (A, B, C ou D). A equipe que acertava, pontuava no placar. Após cada pergunta, os extensionistas abordavam fatores de riscos e causas para as referidas doenças, objetivando que ainda na juventude os alunos possam preveni-las, para viver a velhice da forma mais saudável e com qualidade, deixando como pergunta reflexiva para os mesmos: $\mathrm{O}$ que estou fazendo para prevenir estas doenças? 
De modo a trocar informações sobre as principais patologias em uma pessoa idosa, buscou-se ao final da ação informações sobre quais doenças os idosos que os adolescentes conheciam apresentavam, estimulando a prática da paciência, do cuidado e do zelo com os idosos, entendendo que muitas vezes os mesmos encontram-se limitados à inúmeras atividades não por que optaram, mas pelas dificuldades ao longo do processo de envelhecimento, o qual pode ou não ser acompanhado de patologias.

Tal entendimento ainda na juventude é muito válido e remete a reflexão sobre as decisões e escolhas na alimentação e nos hábitos de vida dos adolescentes, ao ponto que os fazem pensar, nos dias de hoje, em que tipo de idoso eles querem ser, estimulando assim a prática de atividades físicas regulares, uma alimentação saudável e de qualidade, uma juventude e uma fase adulta sem tabagismo e sem o álcool, entre outros fatores que irão, consideravelmente, influenciar na promoção da saúde e na prevenção de doenças na terceira idade.

As respostas dos alunos coincidiram em $100 \%$ com as respostas esperadas pela equipe executora, levando a crer que os mesmos têm consciência das patologias vividas pelos idosos, que se prepararam para a temática e que ficaram atentos ao vídeo explicativo anterior, aprimorando cada vez mais o conhecimento acerca do processo de Envelhecimento.

À medida em que a temática era apresentada aos participantes do Projeto, os extensionistas, esclareciam algumas dúvidas, quando se fazia necessário, sempre com base em material de apoio trabalhado em reuniões de preparo da ação e discutido à luz da literatura atual. Ao longo da ação foram feitos sorteios de brindes para estimular a participação dos alunos. No término da ação entregou-se bombons e convite do próximo encontro, bem como, houve a assinatura da frequência de cada turma e o agradecimento final pela presença de todos.

Além da execução da ação educativa em si, obteve-se como demais resultados:

- $\quad$ Empoderamento de cerca de 200 jovens beneficiados com o Projeto sobre a promoção da saúde e a prevenção de doenças ainda na juventude para vivenciar a velhice da melhor forma possível;

- $\quad$ Promoção de maior crescimento na formação acadêmica e pessoal dos 
discentes e docentes do Curso Técnico em Cuidados de Idosos, IFPB - Mangabeira, equipe executora, mediante discussão e preparo das ações desenvolvidas;

- Estímulo ao Envelhecimento Ativo e Saudável através da busca pelas práticas saudáveis de vida desde a juventude;

- Satisfação positiva entre os envolvidos, tanto por parte da equipe executora como dos jovens beneficiados;

- $\quad$ Adesão de um elevado quantitativo de participantes na Ação;

- Envolvimento da equipe de Professores e Trabalhadores da Escola, favorecendo o estreitamento do vínculo entre equipe executora e beneficiados do Projeto;

- Empoderamento tanto da equipe executora quanto dos jovens beneficiados do Projeto a respeito dos idosos e seu processo de envelhecimento, promovendo uma troca de experiências e maior entendimento da temática.

Por fim, realizou-se uma avaliação, como parte do processo de planejamento/gestão da equipe, visando manter a supervisão, cumprir as metas estabelecidas e, tendo como propósito, identificar problemas potenciais que necessitarão ser aperfeiçoados em futuras ações, elencando os pontos positivos e negativos. Tal discussão se deu de forma livre e aberta para ouvir quaisquer membros da equipe executora.

Além dos registros fotográficos, a ação executada foi registrada integralmente no diário de campo do Projeto de Extensão, o qual contribuiu para a feitura deste manuscrito.

\section{CONCLUSÃO}

Ao final do estudo, percebe-se que o Projeto de Extensão, em questão, por meio da ação realizada 'Principais Doenças que acometem os Idosos' contribuiu de forma significativa enquanto subsídio complementar no entendimento de jovens escolares quanto ao processo de Envelhecimento e as principais doenças que podem está presente na velhice. 
A ação educativa trouxe, de forma geral, impactos positivos para a saúde e qualidade de vida dos participantes, à medida que norteou várias práticas saudáveis a serem implantadas ainda na juventude visando evitar doenças e agravos na velhice, além do aprimoramento dos conhecimentos a cerca da temática trabalhada por meio da troca de saberes e experiências dos envolvidos.

Evidenciou-se o empoderamento de cerca de 200 jovens beneficiados sobre a promoção da saúde ainda na juventude, reforçando a importância do processo educativo como parte fundamental do entendimento de jovens escolares no contexto do Envelhecimento e de como de como prevenir algumas doenças ainda na juventude para vivenciar uma velhice saudável.

Não foram apontadas fragilidades no estudo, pois, para a equipe executora da ação o mesmo significou um espaço de conhecimento, informação e troca de experiências tanto para os beneficiados com o projeto, quanto para os extensionistas.

Reafirma-se com este estudo a importância do processo educativo como parte fundamental do empoderamento de jovens escolares para entender o contexto do Envelhecimento e melhor lidar com as diferenças, patologias e particularidades dos idosos mediante o aumento da expectativa de vida.

Finaliza-se este estudo com o alcance do objetivo estabelecido, e apontando a importante colaboração de Atividades de Educação em Saúde para jovens escolares. É salutar, ressaltar a importância cada vez mais de práticas educativas a respeito do Envelhecimento em outras instituições e repartições, sejam públicas ou privadas, para que cada vez mais se discuta sobre questões voltadas aos ldosos e suas necessidades.

Diante do exposto, espera-se que este estudo traga contribuições para o aprimoramento e desenvolvimento do conhecimento científico sobre a importância da educação em saúde para jovens escolares, tendo como foco o processo de envelhecimento.

Espera-se que este estudo se torne uma ferramenta de divulgação de práticas educativas voltadas para o envelhecimento, incentivando outros estudos a serem desenvolvidos, tendo em vista não só a relevância científica que apresenta, mas também a relevância social diante de todas as informações transmitidas, 
principalmente no que se refere a maneira de se tratar e de se conviver com o idoso na sociedade de hoje.

\section{REFERÊNCIAS BIBLIOGRÁFICAS}

CAMARGOS, M. C. S.; GONZAGA, M. R. Live longer and better? Estimates of healthy life expectancy in the Brazilian population. Cad. Saúde Pública, v. 31, n. 7, p. 1460-1472, 2015.

CAMPOLINA, A. G.; ADAMI, F.; SANTOS, J. L. F.; LEBRÃO, M. L. The health transition and changes in healthy life expectancy in the elderly population: possible impacts of chronic disease prevention. Cad. Saúde Pública, v. 29, n. 6, p. 1217-1229, 2013.

CAPORICCI, S.; NETO, M. F. O. Estudo comparativo de idosos ativos e inativos através da avaliação das atividades da vida diária e medição da qualidade de vida. Motricidade, v. 7, n. 2 , p. 15-24, 2011.

CORDEIRO, J.; CASTILLO, B. L. D.; FREITAS, C. S.; GONÇALVES, M. P. Efeitos da atividade física na memória declarativa, capacidade funcional e qualidade de vida em idosos. Rev. Bras. Geriatr. Gerontol., v. 17, n. 3, p. 541-552, 2014.

LEITE, C. T.; VIEIRA, R. P.; MACHADO, C. A.; QUIRINO, G. S.; MACHADO, M. F. A. S. Prática de educação em saúde percebida por escolares. Cogitare Enferm., v. 19, n. 1, p. 13-19, 2014.

MORAES, E. N. Atenção à Saúde do Idoso: Aspectos Conceituais. Brasília: Organização PanAmericana da Saúde; 2012.

PINTO, M. B.; SANTOS, N. C. C. B.; ALBUQUERQUE, A. M.; RAMALHO, M. N. A.; TORQUATO, I. M. B. Educação em saúde para adolescentes de uma escola municipal: a sexualidade em questão. Cienc Cuid Saude, v. 12, n. 3, p. 587-592, 2013.

QUEIROZ, M.G.D.; FONTOURA, R.A.P.; FRANÇA, D.C.C.; MOI, G.P.; AGUIAR, S.M.H.C.A. Uma análise do acesso dos idosos aos serviços odontológicos no Brasil e os dados dos atendimentos a essa clientela no município de Cuiabá. Connection Online, n. 7, p. 13-24, 2012. 